\title{
Video mosaic for panoramic image
}

\author{
Zhaoxia Fu \\ Party school of shanxi provincial committee of the C.P.C, Taiyuan, 030006, China \\ fzx2005@163.com
}

\begin{abstract}
Keywords: Video mosaic; Multi-resolution analysis; Image registration; Phase correlation Abstract. This paper presents an optimal mosaic method of panoramic video image. Firstly, the multi-resolution decomposition algorithm is applied to image registration, and the registration method of phase correlation is optimized, and the iterative process will be conducted on the image level of low scale. Given the basis of the initial parameters, the frequency of registration iterations of the image level of high scale can decrease, and the registration speed can also accelerate. To some extent, pyramid decomposition avoids the problems of local search algorithm converging to local minimum, and improves convergence performance, and improves the accuracy and robustness of the registration. After obtained the transformation parameters through registration, the original images are carried on the cylindrical projection. Then the morphological fusion from the overlap region is achieved. So it achieves the automatic stitching of an image sequence. Experimental results show that the proposed optimal method has a good real-time mosaic effect.
\end{abstract}

\section{Introduction}

The technology of panoramic video mosaic can be more fully described as a process of scene content through automatically splicing video sequences which have a certain overlap area for panoramic image, and it can compensate for the defects of the low video resolution and small vision field [1]. A variety of methods have been used in the panorama mosaic, but they all have some limitations. Ref. [2] first captures moving target in the video splicing, and then the results are spliced into the foreground, which does not apply to the situation of more complex background. Ref. [3] is based on invariant stitching technique, which is fully automatic and has a good effect. So it is considered to have made a major breakthrough in the image mosaic. But its fusion algorithm is a classical fusion algorithm of multi-resolution spline in image fusion, and it can effectively solve the problem of ghosting in a good effect, and the disadvantage is the higher computational complexity.

A video fragment can be seen as the effect of the image display with a number of frames per second (typically 24 frames per second), and therefore the digital image stitching algorithm can be applied to the video mosaic. Because most video mosaic algorithms have real-time requirements such as video surveillance, military reconnaissance, etc., the video mosaic algorithms should have a faster processing speed. The image mosaic generally includes image preprocessing, image registration and image fusion and other steps, and the success of the image mosaic depends largely on the image registration, which requires the image registration algorithm can have a higher matching accuracy and faster processing speed in the video frame mosaic. In this paper, the multi-resolution decomposition algorithm is applied to the video image registration, which searches for the transformation parameters on a multi-scale decomposition level using phase correlation method. The decomposition algorithm can not only improve the registration speed, but also improve the accuracy of registration by smoothing, reducing the impact of local extreme value. To keep the visual consistency of the actual scene, the registered image is projected on the same cylinder for forming a cylindrical image, and then the overlapping areas are smoothly fused, and finally the cylindrical panorama stitching is completed.

\section{Image registration algorithm}

The registration algorithm of this paper is first to carry on pyramid decomposition in two images preparing for the registration, and then the different resolution images are gotten. Secondly, in the 
lowest scale level of the pyramid, it computes the transformation parameters that are optimal matching with the reference image using the phase correlation method, which is to find the best transformation of two images, i.e., rough registration. Once again the obtained transformation parameters on the lowest scale level are passed to the next level for the higher scale registration as a result of the initial value, i.e., slight registration. In turn until finally the registration results are obtained on the original images.

(1) Multi-resolution decomposition algorithm

The image decomposition of the Gaussian pyramid is as follows [3]. First, the image $G_{l-1}$ whose level is $l-1$ and the window function $w(m, n)$ which has a low-pass characteristic have a convolution, and then the convolution result is sampled by an interval of row and column, i.e.,

$$
G_{l}(i, j)=\sum_{m=-2 n=-2}^{2} \sum^{2} w(m, n) G_{l-1}(2 i+m, 2 j+n) \text { for } 1 \leq l \leq N, 0 \leq i \leq C_{l}, 0 \leq j \leq R_{l}
$$

where $N$ is the level number of the top level of the Gaussian pyramid, and $C_{l}$ is the number of columns of the lth level on the Gaussian pyramid, and $R_{l}$ is the number of rows of the lth level on the Gaussian pyramid.

The $w(m, n)$ is a 5-by-5 window function, as an introduction of reduced operator REDUCE, so the Eq.(1) can be written as

$$
G_{l}=\operatorname{REDUCE}\left(G_{l-1}\right) \text {. }
$$

Thus, $G_{0}, G_{1}, \mathrm{~L}, G_{N}$ constitute the Gaussian pyramid, which $G_{0}$ is the lowest level of the Gaussian pyramid, and $G_{N}$ is the top level of the Gaussian pyramid, and the total number of levels of the Gaussian pyramid is $N+1$.

(2) Motion parameters estimate of Fourier transform

The motion parameters between two adjacent cameras can be obtained rapidly by the phase correlation method using cross-power spectrum [4].

Suppose two separate images $f_{1}$ and $f_{2}$ are relative in the spatial field only with the displacement $\left(x_{0}, y_{0}\right)$, i.e.,

$$
f_{2}(x, y)=f_{1}\left(x-x_{0}, y-y_{0}\right) .
$$

The corresponding Fourier transforms $F_{1}$ and $F_{2}$ will be relative, i.e.,

$$
F_{2}(\varepsilon, \eta)=e^{-j 2 \pi\left(\varepsilon x_{0}+\eta y_{0}\right)} F_{1}(\varepsilon, \eta) .
$$

The cross-power spectrum of two images $f_{1}$ and $f_{2}$ is

$$
\frac{F_{1}^{*}(\varepsilon, \eta) F_{2}(\varepsilon, \eta)}{\left|F_{1}^{*}(\varepsilon, \eta) F_{2}(\varepsilon, \eta)\right|}=e^{-j 2 \pi\left(\varepsilon x_{0}+\eta y_{0}\right)}
$$

where $F_{1}^{*}$ expresses the complex conjugate of $F_{1}$. Carry on the inverse Fourier transform to Eq. (5), and produce function $\delta$ in the place $\left(x_{0}, y_{0}\right)$. After measuring the location of the peak of the inverse Fourier transform of the cross-power spectrum phase about Eq. (5), we will obtain the displacement $\left(x_{0}, y_{0}\right)$ between two image, i.e., 


$$
\delta\left(x-x_{0}, y-y_{0}\right)=F^{-1}\left[e^{-j 2 \pi\left(\varepsilon x_{0}+\eta y_{0}\right)}\right] .
$$

\section{Cylindrical projection model}

After performing image matching, you can put an image projected onto the same cylindrical surface, in order to splice into a panoramic image. The cylindrical projection transformation is divided into two steps: (1) The pixels captured by the camera on the image plane are mapped onto the cylindrical side surface using the pinhole imaging model; (2) Expand the projected region of the cylindrical side surface. The pixels of cylindrical surface are stored in the form of two-dimensional coordinates. According to Ref. [5], the transform equation of cylindrical projection is given by

$$
\left\{\begin{array}{c}
x^{\prime \prime}=R \cdot \arctan \left\{\frac{x-\frac{W}{2}}{R}\right\}+R \cdot \arctan \left(\frac{W}{2 R}\right) \\
y^{\prime \prime}=\frac{R \cdot\left(y-\frac{H}{2}\right)}{\sqrt{\left(x-\frac{W}{2}\right)^{2}+R^{2}}}+\frac{H}{2}
\end{array} .\right.
$$

The fusion of video images using fusion algorithm need be executed for each frame of video, therefore, the fusion algorithm should be simple and effective. Video fusion methods commonly have the weighted average method, the gradual transition methods. However, the two methods will result in the loss of high-frequency information, and the movements of objects in the transition region can cause blurring and ghosting in the splicing results. In order to reduce the impact of ghosting, we use the method based on morphological fusion to fuse the transition region of the image.

\section{Experimental results and analysis}

The Experiments have be executed in the platform of Windows7, Intel (R) Celeron (R) CPU G550@ 2.60GHz, RAM 2 GB. Fig. 1 shows two video images to be stitched, and their sizes all are $331 \times 410$. Fig. 2 compares the traditional stitching result with the mosaic result of this paper. Seen from Fig. 2 (a), the splicing moving targets of the scene have some significant blurs of high frequency content. The result in Fig. 2 (b) is obtained from this mosaic method of the paper, which can effectively suppress the blurs of high frequency content, and the mosaic effect is good.
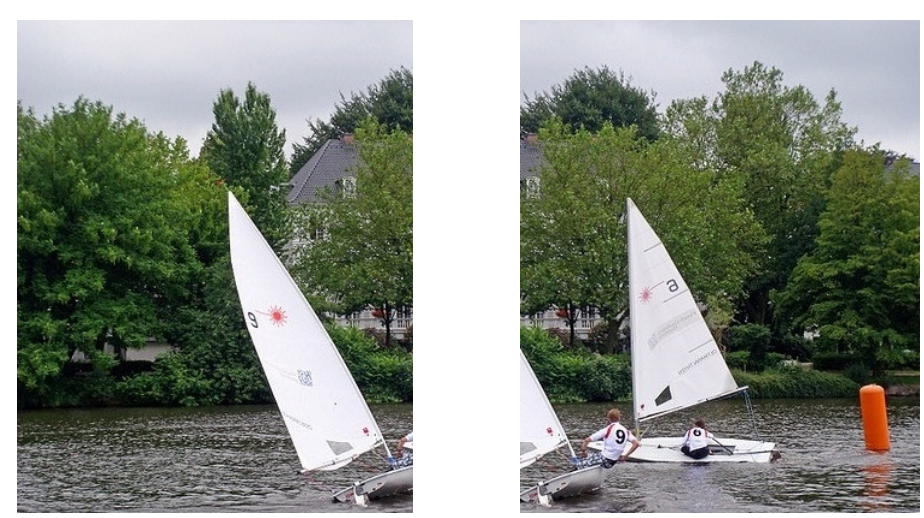

Fig. 1 Original images 


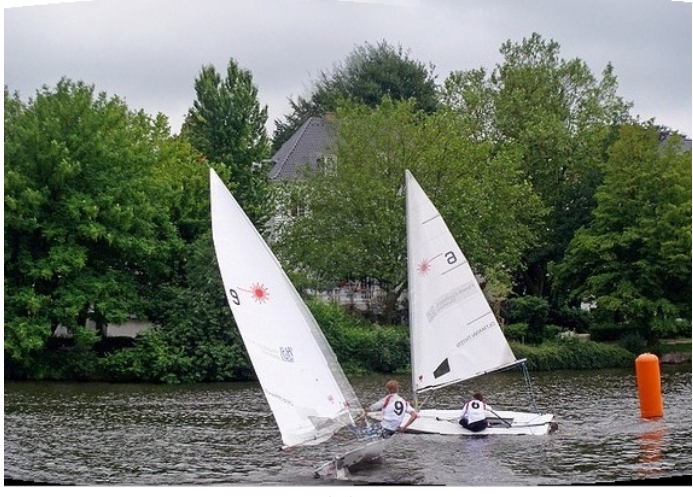

(a)

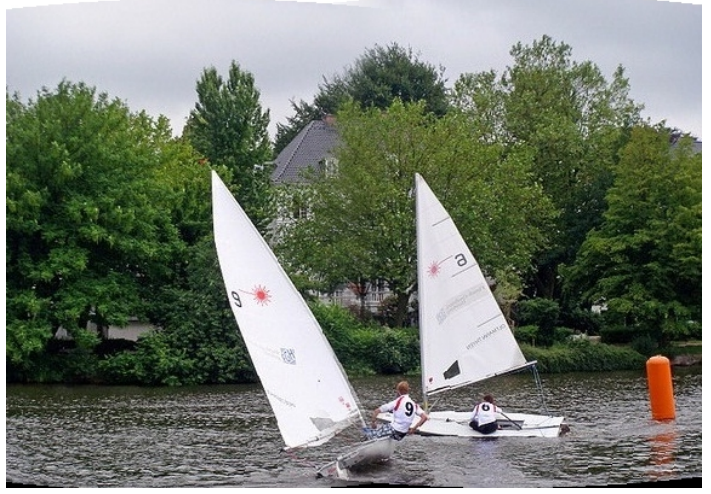

(b)

Fig. 2 Mosaic results: (a) the traditional algorithm, (b) the algorithm of the paper.

\section{Conclusions}

In this paper, the multi-resolution decomposition algorithm is applied to the image registration. In the image registration of low scale, reducing the image size improves the convergence speed in large. By providing initialization estimates that search results are as the initial value of the slighter scale images, it also accelerates the convergence speed of transformation parameters on this scale. Therefore, the use of the multi-resolution decomposition algorithm can not only improve the speed of the registration, but also reduce the influence of local minimum and improve the registration accuracy by smoothing. The simulation experiments show that the proposed optimal method in the paper can obtain the result of image registration accurately and rapidly.

\section{References}

[1] Lin Wu, Hong Jing-xin, Zhang Hao, et a1: Fast and Effective Method for Video Mosaic, Computer Engineering and Applications, Vol.45, issue 24, pp. 173-175. (2009)

[2] Zhang Yuan, Jia Kebin, Liu Pengyu: Video Stitch Algorithm Based on Dynamic Foreground Extraction, Proc. of the 2nd International Congress on Image and Signal Processing, Tianjin, China. (2009)

[3] P.J.Burt, R.J.Kolczynski: Enhanced Image Capture Through Fusion, Proceedings of Fourth International Conference on Computer Vision, issue 5, pp.173-182. (1993)

[4] Kobayashi K, Nakajima H, Aoki T, et al: Principals of Phase Only Correlation and Applications, ITE Technical Report, Vol. 20, issue 41, pp. 1-6. (1996)

[5] Jiang Jing, Liu Tong-ming: Algorithm for Cylindrical Panoramic Image, Journal of East China Shipbuilding Institute (Natural Science Edition), Vol. 18, issue 4, pp. 62-66. (2004) 